\title{
Comprehensive assessment of the disputed RET Y791F variant shows no association with medullary thyroid carcinoma susceptibility
}

\author{
Rodrigo A Toledo', Roxanne Hatakana', Delmar M Lourenço Jr ${ }^{1}$, Susan C Lindsey ${ }^{6}$, \\ Cleber P Camacho ${ }^{6}$, Marcio Almeida ${ }^{9}$, José V Lima Jr ${ }^{10}$, Tomoko Sekiya', \\ Elena Garralda ${ }^{8}$, Michel S Naslavsky ${ }^{4}$, Guilherme L Yamamoto ${ }^{4}$, Monize Lazar ${ }^{4}$, \\ Osorio Meirelles ${ }^{11}$, Tiago J P Sobreira7, Maria Lucia Lebrao ${ }^{3}, Y^{7}$ (a A O Duarte ${ }^{2}$, \\ John Blangero ${ }^{9}$, Mayana Zatz ${ }^{4}$, Janete M Cerutti ${ }^{5}$, Rui M B Maciel ${ }^{6}$ and \\ Sergio P A Toledo ${ }^{1,6}$ \\ ${ }^{1}$ Endocrine Genetics Unit (Laboratório de Investigação Médica/LIM-25) of Hospital das Clínicas, \\ University of São Paulo School of Medicine, São Paulo, São Paulo 05403-010, Brazil \\ ${ }^{2}$ Nursing School, ${ }^{3}$ School of Public Health, and ${ }^{4}$ Human Genome Research Center, University of São Paulo, \\ São Paulo, São Paulo, Brazil \\ ${ }^{5}$ Division of Genetics, Genetic Bases of Thyroid Tumors Laboratory, Department of Morphology and Genetics, and \\ ${ }^{6}$ Division of Endocrinology, Laboratory of Molecular and Translational Endocrinology, Department of Medicine, \\ Federal University of São Paulo, São Paulo, São Paulo, Brazil \\ ${ }^{7}$ Brazilian National Laboratory of Biosciences, Campinas, São Paulo, Brazil \\ ${ }^{8}$ Centro Integral Oncológico Clara Campal, Hospital Universitário Sanchinarro, Madrid, Spain \\ ${ }^{9}$ Department of Genetics, Texas Biomedical Research Institute, AT\&T Genomic Computing Center, \\ San Antonio, Texas, USA \\ ${ }^{10}$ Endocrinology Division, Santa Casa Hospital, São Paulo, São Paulo, Brazil \\ ${ }^{11}$ Laboratory of Epidemiology and Population Sciences, National Institute on Aging, Bethesda, Maryland, USA
}

\author{
Correspondence \\ should be addressed \\ to R A Toledo \\ Emails \\ toledorodrigo@usp.br or \\ toledorodrigo79@gmail.com
}

\begin{abstract}
Accurate interpretation of germline mutations of the rearranged during transfection (RET) proto-oncogene is vital for the proper recommendation of preventive thyroidectomy in medullary thyroid carcinoma (MTC)-prone carriers. To gain information regarding the most disputed variant of RET, ATA-A Y791F, we sequenced blood DNA samples from a cohort of 2904 cancer-free elderly individuals (1261 via Sanger sequencing and 1643 via wholeexome/genome sequencing). We also accessed the exome sequences of an additional 8069 individuals from non-cancer-related laboratories and public databanks as well as genetic results from the Catalogue of Somatic Mutations in Cancer (COSMIC) project. The mean allelic frequency observed in the controls was 0.0031 , with higher occurrences in Central European populations (0.006/0.008). The prevalence of RET Y791F in the control databases was extremely high compared with the 40 known RET pathogenic mutations $(P=0.00003)$, while no somatic occurrence has been reported in tumours. In this study, we report new, unrelated Brazilian individuals with germline RET Y791F-only: two tumour-free elderly controls; two individuals with sporadic MTC whose Y791F-carrying relatives did not show any evidence of tumours; and a 74-year-old phaeochromocytoma patient without MTC. Furthermore, we showed that the co-occurrence of Y791F with the strong RET C634Y mutation explains the aggressive MTC phenotypes observed in a large affected family that was initially reported as Y791F-only. Our literature review revealed that limited analyses
\end{abstract}

http://erc.endocrinology-journals.org DOI: 10.1530/ERC-14-0491 Printed in Great Britain

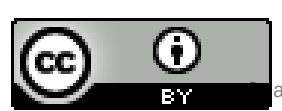

This work is licensed under a Creative Commons Attribution 3.0 Unported License.

\section{Key Words}

- multiple endocrine neoplasias

- RET oncogene

- MEN2

- medullary thyroid carcinoma

- genetics 
have led to the misclassification of RET Y791F as a probable pathogenic variant and, consequently, to the occurrence of unnecessary thyroidectomies. The current study will have a substantial clinical influence, as it reveals, in a comprehensive manner, that RET Y791F only shows no association with MTC susceptibility.

Endocrine-Related Cancer (2015) 22, 65-76

\section{Introduction}

MEN2 (MIM \#164761) is a dominantly inherited multiglandular tumour syndrome that presents with a high penetrance of medullary thyroid carcinoma (MTC; observed in virtually $100 \%$ of cases), phaeochromocytoma (50\%) and parathyroid adenoma and/or hyperplasia (20\%) (Eng et al. 1996). Germline gain-of-function mutations of the rearranged during transfection (RET) proto-oncogene, mostly occurring in exons 8, 10, 11, 13, 14, 15 and 16, activate RET via autophosphorylation of the transmembrane receptor tyrosine kinase; these mutant receptors are highly expressed in MEN2-related tissues, leading to tumour growth. The International Consortium on MTC of 1996 (Eng et al. 1996), the 2001 Consensus on MENs (Brandi et al. 2001) and the updated American Thyroid Association (ATA) Consensus on MTC (Kloos et al. 2009) recommend that individuals carrying a germline disease-causing RET mutation should be subjected to early prophylactic total thyroidectomy, which is currently the only effective approach for preventing the progression of MTC. For the purpose of molecular diagnosis and for adequate surgical management of patients and their relatives, it is crucial to know whether a $R E T$ gene variant identified via genetic testing is a benign polymorphism, a variant of unknown clinical significance or a pathological disease-causing mutation (Toledo et al. 2006).

Biased genetic analyses of highly selected patients, in addition to relatively small numbers of controls, may result in a misunderstanding and misclassification of the pathogenicity of rare variants of medically actionable genes (Weber \& Eng 2005). In this context, there is currently a great debate in the literature regarding the potential pathogenicity of the c.2372A $>$ T Y791F variant in exon 13 of RET (Berndt et al. 1998, Fitze et al. 2002, 2004, Gimm et al. 2002, Brauer et al. 2004, Plaza-Menacho et al. 2007, Tamanaha et al. 2007, Vestergaard et al. 2007, Eng 2010, Erlic et al. 2010, Cerutti \& Maciel 2013, Pęczkowska et al. 2013, Rich et al. 2014).

Recently, we described nine MEN2A families carrying both the classical RET C634Y mutation and the RET Y791F variant, which revealed a previously unrecognised role for
Y791F in the MEN2 syndrome. Although further analysis is still needed, our results indicate that Y791F may modify the effects of other concurrent RET mutations and may therefore act as a modifier variant (Toledo et al. 2010). However, the role of RET Y791F-only in MTC/MEN2related pathogenesis and susceptibility is currently a matter of dispute (Vestergaard et al. 2007, Kloos et al. 2009, Eng 2010, Erlic et al. 2010, Rich et al. 2014 and Supplementary References, see section on supplementary data given at the end of this article).

The current study represents the largest analysis focusing on the RET (ATA-A) Y791F variant performed to date, including new data from Sanger and next-generation sequencing analyses of 2904 healthy adult/elderly individuals. We also accessed genetic and genomic findings from multiple public databases, resulting in the analysis of a total of 11544 healthy individuals and 18163 cancer samples.

\section{Subjects}

Written informed consent was obtained from the subjects, in accordance with the protocols approved by the Institutional Review Board of each participating centre.

\section{Cohorts of new investigated controls}

In this study, we report data from 2904 previously uninvestigated individuals from three tumour-free adult/ elderly cohorts. The first cohort comprised 1261 healthy adult/elderly Brazilians (54\% females and 46\% males), with a mean age of 65.2 years. Blood DNA samples from these individuals were subjected to Sanger sequencing of RET exon 13 in our laboratory at the University of Sao Paulo School of Medicine. This cohort included samples from the School of Public Health of the University of Sao Paulo and control samples from the Experimental Oncology Laboratory (LIM-24) of the University of Sao Paulo School of Medicine. The ethnicity of the tumour-free adult/elderly subjects was 79\% White/White Latino and 10\% African and mixed Black/Caucasian.

Published by Bioscientifica Ltd. 
The second cohort has been designated the SABE60+ cohort and is a control group that was assembled and subjected to whole-exome sequencing by the Human Genome and Stem Cell Research Center (HUG-CELL) of the University of Sao Paulo. SABE60+ consists of 604 Brazilian individuals from Sao Paulo City (214 males and 390 females), with minimum and maximum ages of 63.3 and 92.2 respectively, and an average age of $74.2 \pm 6.9$ years. The majority of the individuals in this cohort were White (57.62\%), followed by mixed Black/Caucasian (18.38\%), Black (7.45\%), Asian (1.99\%) and Amerindian (0.33\%). Approximately 7\% (6.62\%) of the individuals answered positively to belonging to another ethnicity, and $7.62 \%$ did not answer this question.

The third cohort investigated in this study is the T2D-GENES cohort, which comes from a large collaborative study composed of 1039 completely sequenced individuals. The primary focus of this study group is the discovery of new genes associated with the development of type 2 diabetes using the information provided by large human pedigrees. Each sequenced pedigree contains between 22 and 86 individuals distributed across three to five generations. The sample population is composed of Mexican-American (48.9\% males) individuals living in San Antonio, Texas (SATX), who are part of the large San Antonio Family Study (SAFS) project, which has been ongoing for more than 25 years, with a primary focus on cardiovascular diseases.

\section{Public databases}

Genetic and genomic data from the following non-tumourenriched public databases were accessed: i) the NHLBI GO Exome Sequencing Project; ii) the database of single nucleotide polymorphisms (dbSNP) of the National Center for Biotechnology Information (NCBI), National Library of Medicine and iii) Ensembl (European Bioinformatics Institute, EBI, and European Molecular Biology Laboratory, EMBL), from the Wellcome Trust Sanger Institute (WTSI). The genotyping results for the 1000 European-American controls reported by Erlic et al. (2010) were also included.

In addition, genetic and genomic findings from the cancer-specific Catalogue of Somatic Mutations in Cancer (COSMIC) database were also accessed, while variant classifications were obtained from the databases of the ARUP MEN2/RET program of the Department of Pathology, University of Utah (Margraf et al. 2009).

All of the data from the databases were obtained between 20 July and 23 July 2014, and the websites for each database are listed at the end of the article.

\section{Literature review}

The PubMed and Google Scholar databases were used for the literature review. Space limitations prevented us from providing more thorough coverage of the topic, and we apologise to those whose work could not be cited. An extended list of the studies on RET Y791F is included as online supplementary data.

\section{Methods}

\section{DNA extraction, PCR and Sanger sequencing}

Blood DNA was extracted using the salting out method and a Qiagen kit (Qiagen). PCR amplification of RET exon 13 was carried out using $10 \mathrm{ng}$ of gDNA and the following primers: F: $5^{\prime}$-AACTTGGGCAAGGCGATGCA-3' and R: 5'-AGAACAGGGCTGTATGGAGC-3'. The PCR products were run on a $1 \%$ agarose gel, and specific $277 \mathrm{bp}$ amplicons (Supplementary Fig. 3, see section on supplementary data given at the end of this article) were then purified using the ExoSap-IT enzyme (USB Co., Cleveland, $\mathrm{OH}$, USA). BigDye Terminator v.3.1 cycle sequencing (Applied Biosystems) was employed to sequence the reaction products, and the samples were run on an ABI Prism 3130xl - Genetic Analyzer (Applied Biosystems).

\section{Whole-exome and whole-genome sequencing}

Whole-exome sequence data were generated using Agilent SureSelect v2 technology for targeted exon capture, and the obtained reads were aligned using the BurrowsWheeler Aligner (BWH) tool. Piccard was employed to convert, sort and index the aligned data files; the sequence quality scores were recalibrated using Genome Analysis Toolkit (GATK); and annotation of the variants was performed using Annovar.

Whole-genome sequencing (WGS) of 586 individuals was performed externally by Complete Genomics, Inc. (CGI, Mountain View, CA, USA) via a sequence-by-ligation method. The paired-end sequencing reads $(70 \mathrm{bp})$ were mapped using the human reference genome (V 37.2), with a mean coverage of $60 \times$. Variant calling was performed by CGI using version 2.0.3.1 of their proprietary pipeline. The false discovery rate estimates for SNP calls on the CGI platform ranged from 0.2 to $0.6 \%$. The variant calls within the WGS were processed using CGA Tools software (version 1.5.0, build 31), made available by CGI. The WGS information was employed together with the pedigree information and a previously generated genome-wide association chip for effective imputation of

Published by Bioscientifica Ltd. 
offspring using the MACH Bayesian imputation algorithm (Li 2010). The final sample comprised 1039 individuals genotyped using nearly 23 million SNPs.

\section{Protein structure analysis}

The structure of the RET receptor was generated based on the crystal structure of the RET tyrosine kinase domain bound to adenosine that has been recently deposited in the RCSB-PDB protein databank (ID: 4CKJ, Plaza-Menacho et al. 2014) and using the software program YASARA (Krieger et al. 2009). The RET domains were determined using the Conserved Domain Database (CDD), and a figure was generated with DOG 2.0 (Ren et al. 2009).

\section{In silico pathogenic prediction}

Four packages with different algorithms were used to investigate the pathogenicity of RET Y791F in silico: SIFT; PMUT; Align-GVGD; and POLYPHEN2 (the websites are listed at the end of the article).

\section{Statistical analysis}

Statistical analysis was performed by $\mathrm{O}$ Meirelles using IBM SPSS (www-01.ibm.com/software/analytics/spss/).

\section{Results}

\section{RET Y791F in the tumour-free cohorts}

The analyses of 22138 alleles from the control subjects revealed that $\mathrm{Y} 791 \mathrm{~F}$ is a very rare $\mathrm{SNP}$, with a frequency that varies greatly among the analysed populations (Fig. 1 and Supplementary Table 1, see section on supplementary data given at the end of this article). In accordance with an initial

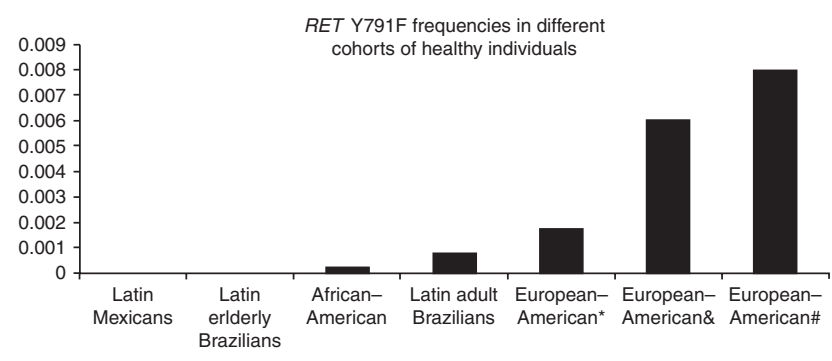

Figure 1

RET Y791F frequencies in different control populations/cohorts. The highest frequencies are observed in the populations from central Europe, especially Germany. Details of the populations are provided in Supplementary Table 1. report from Europe (Berndt et al. 1998), the three EuropeanAmerican cohorts of control individuals presented the highest frequencies, which ranged from 0.0017 to 0.008 . The three Latin cohorts together with the African-American population presented the lowest frequencies of RET Y791F, ranging from 0 to 0.0007 (Supplementary Table 1). Notably, both the original Y791F MTC cases reported by Berndt et al. (1998) and the screen of 1000 controls conducted by Erlic et al. (2010), which identified the highest allelic frequency among the controls, are from Germany. In addition, as discussed later in this study, the largest cohort of patients carrying the Y791F variant also comes from Germany, indicating a probably higher prevalence of the Y791F variant in this country. Interestingly, one of the healthy Y791F-only Brazilian individuals reported in this study and the two unrelated Brazilian cases with sporadic MTC who also carried the variant (descriptions of these individuals are provided later in this report) were direct descendants of Germans. In addition, at least two out of the nine Brazilian MEN2A families with C634Y/Y791F that we had previously reported were of German descent (Toledo et al. 2010, Valente et al. 2013). However, no cases harbouring C634Y/Y791F have been reported outside of Brazil thus far.

Owing to possible incomplete assessment of the phenotypic features of affected individuals or late disease onset, the control databases may conceivably include patients with rare diseases such as (familial) MTC and MEN2 syndrome who carry pathogenic RET variants. To explore the chance of this situation occurring, we assessed whether the Exome Sequencing Project (ESP) exome variation database (which is not a tumour-enriched databank) included any classical disease-associated RET mutations. No alleles corresponding to strong RET mutations, classified as an MTC level-3 risk by the consensus on MENs or as levels C-D by the ATA MTC guidelines, were found in the ESP control databases (Fig. 2 and Supplementary Fig. 1, see section on supplementary data given at the end of this article). Notably, one allele of the low-risk V804M mutation, which is classified as MTC risk 1/ATA-A, was found among the 13000 RET alleles from the ESP database. Valine 804 is a gatekeeper residue that is critical for ATP binding and activation of the RET kinase receptor. Protein conformation analyses have revealed that the V804M and V804L mutations modify the ATP-binding pockets of RET, facilitating ATP binding and conferring resistance to the multikinase inhibitor vandetanib, which is approved for the treatment of advanced MTC (Carlomagno et al. 2004, Wells et al. 2010). The identification of heterozygous RET V804M in the controls may be due to the relatively mild phenotype or late onset of MTC that is frequently associated with this

Published by Bioscientifica Ltd. 


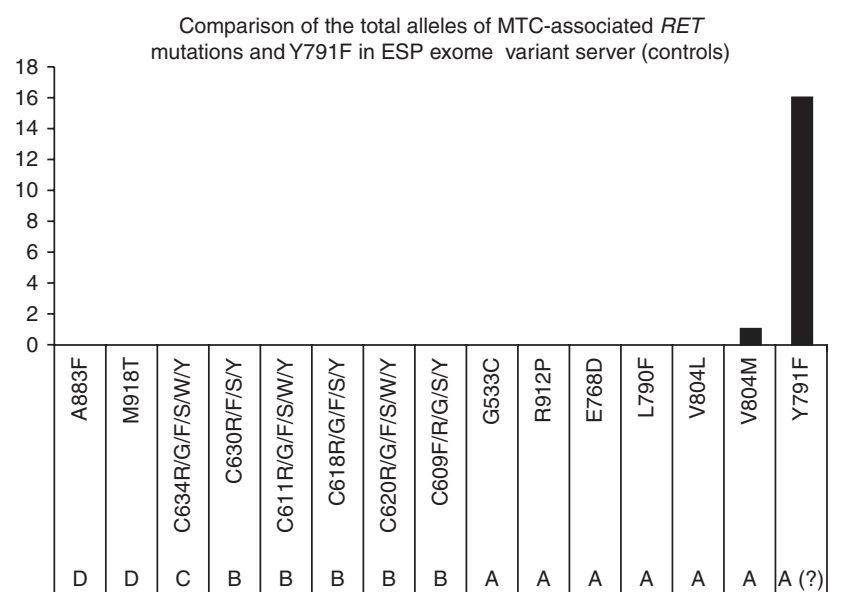

Figure 2

No RET ATA-B, C or D alleles were found in the ESP control databases. Only one instance of the V804M allele (ATA-A) was found among the 13000 sequenced alleles, indicating a very low frequency of bona fide MTCrelated $R E T$ mutations in healthy individuals. In contrast, 15 alleles of $Y 791 \mathrm{~F}$ were found within the ESP control databases, indicating that the frequencies of this specific variant behave differently, possibly as an accompanying genetic modifier or as a benign polymorphism.

variant (Feldman et al. 2000). The ESP dataset also included one allele of each of the three RET variants G321K, K666E, and R866W. These three last variants are very rare and are noted on the ATA guidelines as 'mutations based on limited families/case reports and may represent variants of unknown significance' (Kloos et al. 2009).

In contrast to the absence/very low frequency of bona fide RET-activating mutations in the ESP dataset, the Y791F variant was present in 14 individuals, one of whom was homozygous ( $\mathrm{N}$ alleles $=15$ ). This allelic frequency is significantly higher than that observed among the remaining $R E T$ mutations $(P=0.00003)$.

\section{Previously unreported tumour-free elderly individuals harbouring RET Y791F}

In this study, we report two Brazilian RETY791F carriers who were identified during the genetic screening of the healthy elderly controls by Sanger sequencing performed in our laboratory. The first subject is a 76-year-old woman who has never developed tumours and presents no health complications. She has been followed by the Hospital das Clínicas in Sao Paulo (HC-FMUSP) for routine check-ups. Her calcitonin level was undetectable $(<2 \mathrm{pg} / \mathrm{ml})$ in September 2014, and she reports no history of tumours in her family. Her father was German and died at the age of 75 due to a heart attack. Her 45-year-old daughter (an only child) was invited to come to the hospital to provide blood for biochemical examinations and genetic testing; her calcitonin level was $<2 \mathrm{pg} / \mathrm{ml}$, and sequencing analysis revealed that she harboured the WT allele.

Less information is available regarding the second subject with RET Y791F. She was also followed by HC-FMUSP for routine check-ups, and her medical report shows no tumour development or presence of other serious clinical features. She died in 2008 at the age of 94 .

In accordance with our data on elderly individuals carrying RET Y791F without any history of tumours, a recent study that analysed the exome data of 44 centenarian Ashkenazi Jews has found two individuals $(2 / 44=0.045)$ harbouring the RET Y791F variant (Freudenberg-Hua et al. 2014).

\section{Evaluation of RET Y791F in MTC, MEN2, hyperpara- thyroidism (HPT) and phaeochromocytoma shows no association with susceptibility to endocrine neoplasia}

Berndt et al. (1998) first reported the presence of the Y791F variant in MTC patients in 1998. In addition to the mutational hotspots in exons 10 and 11, the authors reported exon 13 as a new potential hotspot following the identification of the L790F and Y791F variants in patients with familial MTC. In the original paper, the two RET Y791F families were small and included a combined total of only three clinically affected individuals (Berndt et al. 1998). The association linking Y791F to familial MTC/MEN2 is based on limited and selected cohorts. More importantly, there is no convincing data in the literature showing co-segregation of Y791F with MTC (Supplementary References).

Additionally, Gimm et al. (2002) analysed German patients with mutations in codons 790 and 791 and suggested a mild form of the disease.

Through a review of the literature, we found two large families reported to carry RET Y791F; one family is from Denmark with Central Europe descendants, and the other is from Brazil. Vestergaard et al. (2007) initially identified a Danish young case with goitre carrying RET Y791F and subsequently performed genetic screening of a total of 27 family members. It is noteworthy that 15 of the family members were Y791F carriers, and none exhibited abnormal clinical or biochemical results. The calcitonin levels of the carriers and non-carriers in this family did not differ significantly, and total thyroidectomy was postponed (Vestergaard et al. 2007). The second kindred were a large Brazilian RET Y791F family with 17 carriers, ten of whom were diagnosed with MTC, including cases with the early and aggressive phenotype (Tamanaha et al. 2007).

Published by Bioscientifica Ltd. 
Although this family was initially described as an Y791Fonly family, a subsequent analysis indicated that these patients also harboured the bona fide activating germline RET C634Y mutation (Cerutti \& Maciel 2013, Valente et al. 2013). These results are in accordance with the previous report of C634Y-Y791F cis being found in four Brazilian MEN2A families (Toledo et al. 2010).

The largest reported cohort of patients carrying RET Y791F was described by Frank-Raue and colleagues and comprised 22 German MTC/MEN2 cases and 34 screened relatives. The authors concluded that Y791F carriers develop milder clinical features and achieve higher cure rates compared with carriers of the codon 790 and 804 mutations (Frank-Raue et al. 2008). No control samples from the German population were analysed in this study. Patients with Y791F who display hyperparathyroidism and no MTC have been reported as well (Vierhapper et al. 2005). Another study identified RET Y791F in German patients with glioblastoma multiforme and gastric and pancreatic cancers who showed no clinical features of MTC or MEN2 (Rückert et al. 2011).

In accordance with the results of studies by Erlic et al. (2010) and Pęczkowska et al. (2013) showing occasional phaeochromocytoma cases with co-occurrence of the RET Y791F variant and pathogenic variants in other phaeochromocytoma-related genes, we identified a 68 -year-old male with phaeochromocytoma carrying the RET Y791F variant and the new germline variant $\mathrm{D} 236 \mathrm{~N}$ in the $S D H B$ gene. Nevertheless, the pathogenicity of the latter variant is still uncertain.

\section{Two new MTC patients harbouring RET Y791F without a family history of endocrine neoplasias}

In this study, we report a 37-year-old female followed by the MEN clinic of the Federal University of Sao Paulo (UNIFESP) who presented a thyroid nodule with cytological results indicative of Hürthle cell neoplasm and an extremely high calcitonin level $(2079 \mathrm{pg} / \mathrm{ml}$, normal $=8.5 / 5.0 \mathrm{pg} / \mathrm{ml})$. During physical examination, the patient showed no cutaneous lichen amyloidosis, marfanoid habitus or mucosal neuromas. She underwent total thyroidectomy with prophylactic central neck dissection. A pathological analysis revealed a $2.5 \mathrm{~cm}$ MTC on the left thyroid lobe and lymphocytic thyroiditis with clusters of hyperplastic Hürthle cells. The tumour was confined to the thyroid, and the 20 lymph nodes that were analysed showed no evidence of metastasis. A genetic analysis of all of the RET hotspot exons revealed the $Y 791 \mathrm{~F}$ variant. Three years after surgery, the calcitonin levels of this patient remain undetectable $(<2 \mathrm{pg} / \mathrm{ml})$; her carcinoembryonic antigen (CEA) level was $1.6 \mathrm{ng} / \mathrm{ml}$, and cervical ultrasonography showed no sign of relapse. Additionally, her clinical and biochemical screening results were negative for phaeochromocytoma and hyperparathyroidism.

Screening of this patient's family revealed no history of endocrine tumours. Her 32-year-old sister also carries the RET Y791F variant and presented a thyroid ultrasound exhibiting thyroiditis and no nodules and biochemical examinations showing no evidence of MTC (calcitonin levels $<1 \mathrm{pg} / \mathrm{ml}$ ) or primary hyperparathyroidism. She was followed by another service and inadvertently underwent thyroidectomy. Pathological analysis found no evidence of MTC or C-cell hyperplasia. Her mother (59 years old) and her two sons (18 and 11 years old) did not harbour the RET Y791F variant and presented low calcitonin levels. Her father, who was probably the obligatory carrier, died in a car accident at the age of 46 years with no signs of the disease. He was from Germany. The lack of a family history of MTC in this family with three carriers argues that the RET Y791F variant is not an endocrine-neoplasia-susceptibility variant. Unfortunately, similar to this family, other asymptomatic relatives with the Y791F variant have also undergone unnecessary thyroidectomies (Supplementary References).

Recently, we have identified another patient with MTC harbouring the RET Y791F variant from the city of Curitiba, Brazil. The patient, a 35-year-old woman, was diagnosed with a thyroid nodule. The patient underwent a thyroidectomy, and the final histological analysis revealed MTC. An extended RET analysis (exons 1-12 and 14-21) was performed, and no other mutations were identified. The patient has a 9-year-old daughter exhibiting normal thyroid ultrasound results. The patient also has a German background.

\section{A new elderly phaeochromocytoma patient harbouring RET Y791F without MTC}

A RET Y791F germline variant was observed in a patient being followed at Santa Casa Hospital in Sao Paulo, who was referred to our laboratory for genetic testing at the University of Sao Paulo. The remaining RET hotspot exons were sequenced, and no variant was identified. The patient was a 74-year-old man diagnosed with a left adrenal incidentaloma $(9 \times 8.3 \times 6.5 \mathrm{~cm})$. He had shown hypertension for the past 14 years, in addition to exhibiting chronic atrial fibrillation and subclinical autoimmune hyperthyroidism. His surgical pathology was diagnostic of phaeochromocytoma $(8.5 \mathrm{~cm}$ and $220 \mathrm{~g})$, and the results

Published by Bioscientifica Lto. 
of his thyroid ultrasound examination were compatible with thyroiditis without nodules. His calcitonin and calcium/parathyroid hormone levels were normal. No family history of tumours was reported, and the sequencing results for the TMEM127 gene showed no mutations. This case is another example of an elderly individual harbouring the RET Y791F variant without any increased risk for MTC.

\section{RET Y791F in human tumours}

The number of RET ATA mutations observed in the COSMIC genetic and genomic dataset increased substantially according to the mutation's aggressiveness: $94.1 \%$ of the ATA RET mutations occurring in cancers belong to the ATA C-D classification (strong mutations), while only 1.4\% are weak ATA-A RET mutations (Fig. 3 and Supplementary Tables 2 and 3 , see section on supplementary data given at the end of this article). Among the ATA-A risk mutations, the most common variant is $\mathrm{V} 804 \mathrm{M}$, while the remaining variants are very rare and with an uncertain functional effect according to the ATA classification.

Among the entire COSMIC dataset of 18163 tumour samples with available RET genotypes, only one (a lymphoid neoplasm, ID: COSM1159820) presented the RET Y791F variant $(0.002 \%$ - no information available

\begin{tabular}{|c|c|}
\hline ATA classification & RET mutations on COSMIC \\
\hline ATA-A & $8(1.4 \%)$ \\
\hline ATA-B & $26(4.5 \%)$ \\
\hline ATA-C & $64(11.2 \%)$ \\
\hline ATA-D & $474(82.9 \%)$ \\
\hline Total & 572 \\
\hline
\end{tabular}

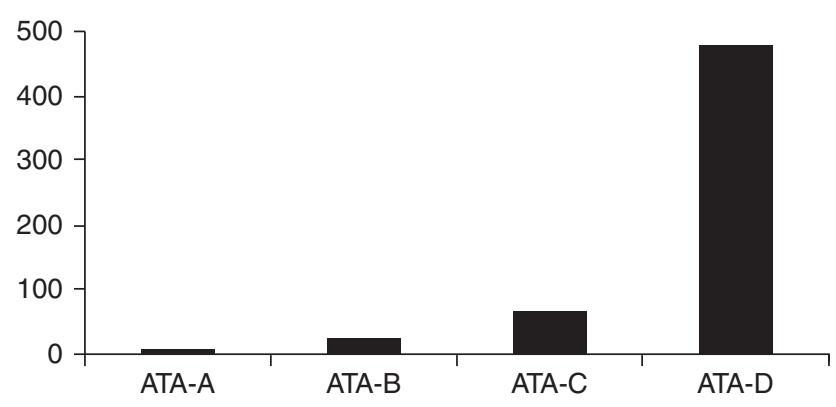

Figure 3

The number of RET mutations observed in the COSMIC cancer genetic and genomic dataset increases substantially according to the ATA mutation aggressiveness classification: $94.1 \%$ of the RET mutations found in cancers belong to the ATA C-D classification (strong mutations), while only $1.4 \%$ consist of weak ATA-A RET mutations. One of the $8(12.5 \%)$ ATA-A variants is $Y 791 \mathrm{~F}$, corresponding to $0.17 \%$ of the mutations. regarding somatic or germline status). Of the 1549 MTCs in the COSMIC dataset, 625 (40.3\%) exhibited a RET mutation, none of which were Y791F, indicating that there is not a frequent association between this variant and MTC tumourigenesis (Supplementary Table 4, see section on supplementary data given at the end of this article).

In addition to the lack of evidence regarding a 'driver' property of RET Y791F in human tumours, two reports describing results indicative of a 'passenger' role for Y791F. The first case is an Y791F MTC patient in whom the most aggressive RET mutation, M918T, was identified somatically, which is most probably the cause of MTC (Fitze et al. 2002). The second case has been recently reported by Carlino et al. (2014) who described a patient with pancreatic carcinoma presenting the typical KRAS G12D mutation and the RET Y791F variant. There is no information as to whether the Y791F mutation was germline or somatic in this patient, but as KRAS G12D is a very well-described pancreatic cancerdriver mutation, the results strongly indicate a minor/ non-pathological role for RET Y791F. Interestingly, the patient is Caucasian, was born in Australia and has a German surname (Carlino, electronic communication).

\section{RET Y791F in HSCR}

RET Y791F was first described in a case of Hirschsprung's disease (HSCR) (Seri et al. 1997). HSCR is a genetically heterogeneous disorder, and inactivation (nonsense and frameshift) mutations in RET are its primary cause (Edery et al. 1994). In families harbouring activating RET mutations occurring primarily in extracellular domains (i.e. exon 10), such as C620R, it has been classically reported that HSCR develops in up to $7 \%$ of carriers. Since the initial report, other Y791F-carrier HSCR cases with no evidence of MTC have been reported, raising the question as to whether this variant is actually involved in tumourigenesis (Virtanen et al. 2013).

\section{In silico RET Y791F analyses}

All four of the software programs used in this study predicted low or very low pathogenicity for the RET Y791F variant (data not shown). Although the software programs employ different algorithms to calculate potential pathogenicity, they all use evolutionary conservation, which indicated that an amino acid change at Tyrosine 791 is more likely to be tolerated, rather than being lethal or pathogenic. Our assessment of the RET protein structure indicate that $\mathrm{Y791F}$ localises to the N-lobe, in $\beta 4$, and is close to the ATP-binding site (Supplementary Fig. 3),

Published by Bioscientifica Ltd. 
which is a region that does not commonly contain strong RET mutations.

\section{In vitro RET Y791F assays}

Plaza-Menacho et al. (2007) investigated the RET Y791F variant in vitro and reported ligand-independent activation. According to the results of this previous study, Y791F is an autophosphorylated monomeric activated receptor that leads to the constitutive activation of STAT3. However, the mechanism of STAT3 activation occurs via Src, JAK1 and JAK2, which differs from the classical RET C634R activation mechanism (Plaza-Menacho et al. 2007). In a later study, Hyndman et al. (2013) analysed in vitro RET variants that had been previously reported in HSCR cases and showed that Y791F behaved similarly to the WT protein in many respects, with similar RET/pERK/pSTAT3 phosphorylation, surface localisation, and RNA and protein stability being observed. Nevertheless, unlike to the RET WT protein and similarly to other HSCR variants, Y791F was associated with reduced levels of cell migration and no ability to promote colony formation (Hyndman et al. 2013). Although this last study did not include bona fide RETactivating mutations for comparison, the results indicate WT-like functional and molecular effects of Y791F, rather than Y791F acting as a gain-of-function mutation.

A third study by Cosci et al. (2011) combined in silico and in vitro assays to analyse known pathogenic mutations of RET, such as C634R and M918T and 'rare medium/lowrisk' variants, including Y791F. Correlations between the results obtained using different approaches were observed primarily for the stronger variants, while the overall results and classification of the weaker variants remained unclear. The in silico classification of RET Y791F was deleterious, although focus and soft agar assays indicated Y791F to be a weak and probably non-pathogenic variant (Cosci et al. 2011). In contrast, the high proliferation rates associated with Y791F, C634R and M918T achieved statistical significance, but these results were inconclusive, as several probably non-pathogenic variants, such as V648I, also achieved statistical significance.

The three studies of RET cited above, together with many others in the literature, show how difficult and sometimes capricious it can be to characterise a weak variant using in vitro or in silico approaches, especially in genes related to genotypes with major medical implications, such as the recommendation for total thyroidectomy in the case of RET. To the best of our knowledge, there is no animal model of the RET Y791F variant.

\section{RET Y791F as a potential phenotypic modifying variant in cases with pathogenic $R E T$ mutations}

The data that we were able to generate and review in the current study indicate a clear lack of pathogenicity of RET Y791F-only. However, the possible role of Y791F as a phenotype-modifying variant cannot be discarded. In fact, we have recently reported four MEN2A families carrying the classical RET C634Y mutation and Y791F in cis (Toledo 2010). The patients presented aggressive MTC (codon C634-like pattern) associated with an increased susceptibility to unusually large bilateral phaeochromocytomas. This finding is indicative of a modifier effect of $\mathrm{Y791F}$ when associated with classical disease-causing RET mutations (Toledo 2010). In the same study, we described in vitro analyses performed in Dr Lois Mulligan's laboratory (ON, Canada), demonstrating that the double C634Y/ Y791F RET receptor is significantly more highly phosphorylated than either the WT receptor or the single C634Y and Y791F RET mutants (Toledo et al. 2010). Interestingly, an association between RET C634Y and Y791F has been recently observed in five additional Brazilian families (Cerutti \& Maciel 2013, Valente et al. 2013). In addition to $\mathrm{C} 634 \mathrm{Y} / \mathrm{Y} 791 \mathrm{~F}$, the presence of $\mathrm{Y791F}$ has been reported in patients harbouring other disease-associated RET mutations, such as C620F, V804L and M918T (Jindrichova et al. 2004). Although current knowledge shows no relevant clinical effect, the possible mechanism of such co-occurrence seems worth exploring in the future.

\section{Known RET missense variants identified through whole-exome/genome sequencing}

Four variants classified as ATA-A mutations (S649L, V804M, R844Q and S891A) (Kloos et al. 2009) were rarely found in the whole-exome/genome sequences of the tumour-free individuals assessed in the current study. The V804M and S891A variants are also included in the RET list as MTC level 1 mutations (Brandi et al. 2001). As mentioned earlier, V804M is a well-described gatekeeper mutation associated with a mild, late-onset phenotype, which could explain its rare occurrence in the presumably healthy cohort (1/13000 alleles in the ESP dataset, 0.00007). The frequencies of V804M and Y791F in the controls were very different and were compatible with a true, low-risk mutation and a rare non-pathogenic variant respectively (Fig. 2 and Supplementary Fig. 2, see section on supplementary data given at the end of this article). In addition, a novel change involving Valine 804 (c.2411C > T; p.V804A) was, to the best of our knowledge, identified

Published by Bioscientifica Ltd. 
Table 1 The nine new variants of the RET proto-oncogene identified via whole-exome and whole-genome sequencing in the current study

\begin{tabular}{|c|c|c|c|}
\hline Casuistic & Nucleotide & Codon & RET protein \\
\hline SABE60+ & C1352T & 451 & T451M* \\
\hline SATX & T1418A & 473 & L473Q \\
\hline SATX & A1462T & 488 & T488S \\
\hline SABE60+ & C1573T & 525 & R525W \\
\hline SABE60+ & C1678T & 560 & P560S \\
\hline SABE60+ & G1799T & 600 & R600L \\
\hline SATX & C2077T & 693 & R693C \\
\hline SABE60+ & C2225T & 742 & T742M \\
\hline SABE60+ & A2255G & 752 & Y752C \\
\hline SATX & T2411C & 804 & V804A \\
\hline
\end{tabular}

\begin{tabular}{lcc} 
dbSNP or ESP & & SIFT \\
\cline { 1 - 1 } Not reported & & D \\
Not reported & & D \\
Not reported & T \\
Not reported & D \\
Not reported & D \\
Not reported & D \\
Not reported & D \\
Not reported & D \\
Not reported & T \\
Not reported & D \\
\hline
\end{tabular}

\begin{tabular}{c} 
PolyPhen2 \\
\hline B \\
D \\
B \\
D \\
D \\
B \\
D \\
D \\
D \\
D
\end{tabular}

\begin{tabular}{ll}
\hline $\boldsymbol{n}$ \\
\hline 1 \\
3 \\
1 \\
1 \\
1 \\
1 \\
2 \\
1 \\
1 \\
1
\end{tabular}

\begin{tabular}{c}
\hline $\begin{array}{c}\text { Genotype } \\
\text { frequency }\end{array}$ \\
\hline 0.0008 \\
0.0014 \\
0.0004 \\
0.0008 \\
0.0008 \\
0.0008 \\
0.0001 \\
0.0008 \\
0.0008 \\
0.0005
\end{tabular}

Please see the discussion and interpretation of these findings in the main text. The T451M variant is not present in the control databases (dbSNP and ESP), but it was identified in two endometrium carcinomas (COSMIC project IDs: 1584958 and 918112). The other variants have not been reported in COSMIC. D, damaging; B, benign; T, tolerated; SABE60 +, University of Sao Paulo; SATX, San Antonio, Texas.

for the first time in this study (Table 1, allelic frequency of 0.00001 in the SATX control cohorts). An in silico analysis using both SIFT and Polyphen2 indicated the V804M and V804A changes to be pathogenic.

In accordance with our results for S649L, seven individuals from the ESP dataset were also identified as harbouring this variant, which has been presumably associated with late-onset, non-aggressive MTC (ColomboBenkmann et al. 2008).

RET V292M was also found in the SABE60+ cohort ( 1 out of 1218 of the sequenced alleles, 0.0008). This variant is classified as pathogenic in the ARUP MEN2 and $\mathrm{dbSNP} / \mathrm{NCBI}$ databases, but it has not been included in the MTC guidelines (Eng et al. 1996, Kloos et al. 2009). Currently, limited evidence is available regarding the pathogenicity of this variant.

The previously reported RET Y791N variant was identified in two control individuals analysed via Sanger sequencing $(2 / 2522,0.008)$ : a 76 -year-old male and a 55 -year-old female. There is no evidence of pathogenicity of this variant in the literature; it was initially reported in a case of HSCR and later in the sister of an MTC case harbouring RET R770Q (Ruiz-Ferrer et al. 2006, Frank-Raue et al. 2010).

\section{Novel RET missense variants identified via whole-exome/genome sequencing}

Nine of the RET missense variants identified herein via massive parallel DNA sequencing have not been reported in the dbSNP/NCBI or ESP databanks and, to the best of our knowledge, are novel (Table 1). Eight of the variants were classified as damaging by Polyphen 2 or SIFT; six were classified as damaging by both software programs; and one variant was classified as benign/tolerated by both software programs (Table 1). Despite the rarity of these variants and the in silico predictions, the fact that our population was composed of healthy adult/elderly individuals with no familial history of tumours indicates that these variants are probably benign polymorphisms or lowpenetrance genetic changes. If new evidence appears in the future indicating pathogenicity in these variants, especially V804A, which occurs at a known RET hotspot, we will contact the carriers and offer genetic counselling, molecular testing and specific clinical screening.

\section{Conclusions of the current comprehensive assessment of RET Y791F}

1. There is no robust evidence from either our data or the literature indicating that RET Y791F-only may cause familial MTC or MEN2 syndrome (reports addressing very small families should not be considered to be informative). RET Y791F, like other non-pathogenic variants of RET, can be found in sporadic MTC patients, but its presence alone does not indicate an increased MTC risk.

2. The frequencies of the RET Y791F variant detected in large cohorts of healthy individuals are characteristic of the behaviour of a rare benign polymorphism, rather than a medically actionable pathogenic mutation of the RET proto-oncogene.

Lessons from findings 1 and 2:

- Asymptomatic germline RET Y791F-only carriers (with no other disease-causing mutations of RET) should not undergo preventive total thyroidectomy

Published by Bioscientifica Ltd. 
based on the genetics. Familial risk, clinical outcomes, and biochemical and imaging data are the most valuable resources for managing these cases.

- There is no indication that genetic and clinical screening of family members of typical sporadic MTC cases harbouring RET Y791F-only is beneficial.

3. The patients with familial MTC/MEN2 who were initially reported to be RET Y791F-only carriers were also found to harbour other associated disease-causing RET variants (C634Y in cis), leading to the disease.

Lessons from finding 3 :

- Genetic testing for RET is only complete when sequencing of all of the hotspot mutations in exons 8 and 10-16 is successfully performed. That is, sequencing of the remaining exons should not cease when the first suspect variant is identified.

- Sequencing or re-sequencing of the hotspot exons of the RET gene (and possibly the full gene depending on the phenotype) of familial MTC/MEN2 patients initially described as RET Y791F-only carriers can reveal medically actionable pathogenic mutations in the RET proto-oncogene.

- Limitations of PCR, such as allelic drop-out (PCR failure of one allele) and/or preferential amplification (hypo-amplification of one allele), may be responsible for false-negative RET genetic results. Therefore, the use of a second set of primers (with different sequences than those used for the initial PCR and sequencing run) is recommended for the re-sequencing of questionable cases.

4. Phaeochromocytoma patients initially reported to be RET Y791F-only carriers can harbour variants of other genes, e.g. VHL or SDHx, that are most probably the cause of the disease.

Lesson from finding 4 :

- Established clinical predictors and algorithms should be used to sequence phaeochromocytomasusceptibility genes in phaeochromocytoma cases that are initially reported as RET Y791F-only.

5. Biased genetic analysis of highly selected patients and a relatively small number of controls can result in misunderstanding and misclassification of the pathogenicity of rare variants in medically actionable genes. Lesson from finding 5 :

- The analysis of large and informative datasets from tumour-free individuals constitutes an accessible and useful tool for improving the interpretation of a molecular diagnosis and aiding in genetic counselling and the clinical management and treatment of carriers.

\section{Final comments about the era of NGS}

The scientific and medical community working on RETrelated diseases needs to be aware of the implications related to the dramatic increase in the number of new variants of this gene being identified in the current nextgeneration sequencing era. In fact, nine new RET variants were identified in the present study. Moreover, it is imperative to use caution when interpreting genetic variants with limited information. At the same time, access to genetic data from large cohorts presents an opportunity to re-evaluate the clinical behaviour of disputed RET variants, such as Y791F.

\section{Websites}

1. NHLBI ESP Exome Sequencing Project: http://evs.gs. washington.edu.

2. dbSNP/NCBI: http://www.ncbi.nlm.nih.gov/SNP.

3. Ensembl EBI/EMBL/WTSI: http://www.ensembl.org.

4. COSMIC: http://cancer.sanger.ac.uk/cancergenome/ projects/cosmic.

5. ARUP RET database, Univ. Utah: http://arup.utah. edu/database/MEN2/MEN2_display.php.

6. SIFT: sift.jcvi.org.

7. PMUT: http://mmb2.pcb.ub.es:8080/PMut/.

8. POLYPHEN2: http://genetics.bwh.harvard.edu/pph2/.

9. Align-GVGD: http://agvgd.iarc.fr/.

Supplementary data

This is linked to the online version of the paper at http://dx.doi.org/10.1530/ ERC-14-0491.

Declaration of interest

The authors declare that there is no conflict of interest that could be perceived as prejudicing the impartiality of the research reported.

\section{Funding}

This study was supported by funds from the Conselho Nacional de Desenvolvimento Científico e Tecnológico (CNPq), grant number 401.990/2010-9 and the Fundação de Amparo à Pesquisa do Estado de São Paulo (FAPESP) grants number 2013/01476-9 (to S P A Toledo) and 2006/60402-1 and 2010/51547-1 (to R M B Maciel). S P A Toledo, J M Cerutti and R M B Maciel are investigators with the CNPq. R A Toledo has received a Ciências Sem Fronteiras CNPq postdoctoral fellowship and is currently working at the Centro Nacional de Investigaciones Oncológicas (CNIO) in Madrid. R Hatakana, T Sekiya, S C Lindsey, D M Lourenço Jr, and R A Toledo received research fellowships from FAPESP. SPA Toledo is a Senior Professor in the Division of Endocrinology, Department of Medicine, Federal University of São Paulo, SP, Brazil, with a grant from the Programa

Published by Bioscientifica Ltd. 
de Visitante Nacional Senior - Edital 028/2013 - from the Coordenadoria de Aperfeiçoamento de Pessoal de Nível Superior (CAPES), Brazil.

\section{Acknowledgements}

The authors would like to acknowledge all of the subjects and volunteers who participated in this study. They would also like to thank Dr Roger Chammas (Experimental Oncology Laboratory/LIM-24, University of Sao Paulo School of Medicine) and Dr Patricia L Dahia (University of Texas Health Science Center at San Antonio) for their support.

\section{References}

Berndt I, Reuter M, Saller B, Frank-Raue K, Groth P, Grussendorf M, Raue F, Ritter MM \& Höppner W 1998 A new hot spot for mutations in the ret protooncogene causing familial medullary thyroid carcinoma and multiple endocrine neoplasia type 2A. Journal of Clinical Endocrinology and Metabolism 83 770-774. (doi:10.1210/jcem.83.3.4619)

Brandi ML, Gagel RF, Angeli A, Bilezikian JP, Beck-Peccoz P, Bordi C, Conte-Devolx B, Falchetti A, Gheri RG, Libroia A et al. 2001 Guidelines for diagnosis and therapy of MEN type 1 and type 2 . Journal of Clinical Endocrinology and Metabolism 86 5658-5671. (doi:10.1210/jcem.86.12.8070)

Brauer VF, Scholz GH, Neumann S, Lohmann T, Paschke R \& Koch CA 2004 RET germline mutation in codon 791 in a family representing 3 generations from age 5 to age 70 years: should thyroidectomy be performed? Endocrine Practice 10 5-9. (doi:10.4158/EP.10.1.5)

Carlino MS, Kwan V, Miller DK, Saunders CA, Yip D, Nagrial AM, Tomlinson J, Grimmond SM, Scolyer RA, Kefford RF et al. 2014 New $R A S$-mutant pancreatic adenocarcinoma with combined BRAF and MEK inhibition for metastatic melanoma. Journal of Clinical Oncology (In Press). (doi:10.1200/JCO.2013.51.5783)

Carlomagno F, Guida T, Anaganti S, Vecchio G, Fusco A, Ryan AJ, Billaud M \& Santoro M 2004 Disease associated mutations at valine 804 in the RET receptor tyrosine kinase confer resistance to selective kinase inhibitors. Oncogene 23 6056-6063. (doi:10.1038/sj.onc.1207810)

Cerutti JM \& Maciel RM 2013 An unusual genotype-phenotype correlation in MEN 2 patients: should screening for RET double germline mutations be performed to avoid misleading diagnosis and treatment? Clinical Endocrinology 79 591-592. (doi:10.1111/cen.12155)

Colombo-Benkmann M, Li Z, Riemann B, Hengst K, Herbst H, Keuser R, Gross U, Rondot S, Raue F, Senninger N et al. 2008 Characterization of the RET protooncogene transmembrane domain mutation S649L associated with nonaggressive medullary thyroid carcinoma. European Journal of Endocrinology 158 811-816. (doi:10.1530/EJE-07-0817)

Cosci B, Vivaldi A, Romei C, Gemignani F, Landi S, Ciampi R, Tacito A, Molinaro E, Agate L, Bottici V et al. 2011 In silico and in vitro analysis of rare germline allelic variants of RET oncogene associated with medullary thyroid cancer. Endocrine-Related Cancer 18 603-612. (doi:10.1530/ERC-11-0117)

Edery P, Lyonnet S, Mulligan LM, Pelet A, Dow E, Abel L, Holder S, NihoulFékété C, Ponder BA \& Munnich A 1994 Mutations of the RET protooncogene in Hirschsprung's disease. Nature 367 378-380. (doi:10.1038/ $367378 \mathrm{a} 0)$

Eng C 2010 Mendelian genetics of rare - and not so rare - cancers. Annals of the New York Academy of Sciences 1214 70-82. (doi:10.1111/j.1749-6632. 2010.05789.x)

Eng C, Clayton D, Schuffenecker I, Lenoir G, Cote G, Gagel RF, van Amstel HK, Lips CJ, Nishisho I, Takai SI et al. 1996 The relationship between specific RET proto-oncogene mutations and disease phenotype in multiple endocrine neoplasia type 2. International RET mutation consortium analysis. Journal of the American Medical Association 276 1575-1579. (doi:10.1001/jama.1996.03540190047028)
Erlic Z, Hoffmann MM, Sullivan M, Franke G, Peczkowska M, Harsch I, Schott M, Gabbert HE, Valimäki M, Preuss SF et al. 2010 Pathogenicity of DNA variants and double mutations in multiple endocrine neoplasia type 2 and von Hippel-Lindau syndrome. Journal of Clinical Endocrinology and Metabolism 95 308-313. (doi:10.1210/jc.2009-1728)

Feldman GL, Edmonds MW, Ainsworth PJ, Schuffenecker I, Lenoir GM, Saxe AW, Talpos GB, Roberson J, Petrucelli N \& Jackson CE 2000 Variable expressivity of familial medullary thyroid carcinoma (FMTC) due to a RET V804M (GTG $\rightarrow$ ATG) mutation. Surgery 128 93-98. (doi:10.1067/msy.2000.107103)

Fitze G, Schierz M, Bredow J, Saeger HD, Roesner D \& Schackert HK 2002 Various penetrance of familial medullary thyroid carcinoma in patients with RET protooncogene codon 790/791 germline mutations. Annals of Surgery 236 570-575. (doi:10.1097/00000658-200211000-00006)

Fitze G, Saeger HD, Roesner D \& Schackert HK 2004 Management of multiple endocrine neoplasia syndrome type 2 families in association with rare germline mutations of the RET proto-oncogene. Klinische Pädiatrie 216 270-276. (doi:10.1055/s-2004-44902)

Frank-Raue K, Machens A, Scheuba C, Niederle B, Dralle H, Raue F \& German MEN2 Study Group 2008 Difference in development of medullary thyroid carcinoma among carriers of RET mutations in codons 790 and 791. Clinical Endocrinology(Oxford) 69 259-263. (doi:10.1111/j.1365-2265.2008.03215.x)

Frank-Raue K, Döhring J, Scheumann G, Rondot S, Lorenz A, Schulze E, Dralle H, Raue F \& Leidig-Bruckner G 2010 New mutations in the RET protooncogene-L881V - associated with medullary thyroid carcinoma and -R770Q - in a patient with mixed medullar/follicular thyroid tumour. Experimental and Clinical Endocrinology \& Diabetes 118 550-553. (doi:10.1055/s-0029-1241851)

Freudenberg-Hua Y, Jan Freudenberg J, Vacic V, Abhyankar A, Emde AK, Ben-Avraham D, Barzilai N, Oschwald D, Christen E, Koppel J et al. 2014 Disease variants in genomes of 44 centenarians. Molecular Genetics \& Genomic Medicine 2 438-450. (doi:10.1002/mgg3.86)

Gimm O, Niederle BE, Weber T, Bockhorn M, UkkatJ, Brauckhoff M, Thanh PN, Frilling A, Klar E, Niederle B et al. 2002 RET proto-oncogene mutations affecting codon 790/791: a mild form of multiple endocrine neoplasia type 2A syndrome? Surgery 132 952-959. (doi:10.1067/msy. 2002.128559)

Hyndman BD, Gujral TS, Krieger JR, Cockburn JG \& Mulligan LM 2013 Multiple functional effects of RET kinase domain sequence variants in Hirschsprung disease. Human Mutation 34 132-142. (doi:10.1002/ humu.22170)

Jindrichova S, Vcelak J, Vlcek P, Neradilova M, Nemec J \& Bendlova B 2004 Screening of six risk exons of the RET proto-oncogene in families with medullary thyroid carcinoma in the Czech Republic. Journal of Endocrinology 83 257-265. (doi:10.1677/joe.1.05838)

Kloos RT, Eng C, Evans DB, Francis GL, Gagel RF, Gharib H, Moley JF, Pacini F, Ringel MD, Schlumberger M et al. 2009 Medullary thyroid cancer: management guidelines of the American Thyroid Association. Thyroid 19 565-612. (doi:10.1089/thy.2008.0403)

Krieger E, Joo K, Lee J, Lee J, Raman S, Thompson J, Tyka M, Baker D \& Karplus K 2009 Improving physical realism, stereochemistry, and sidechain accuracy in homology modeling: four approaches that performed well in CASP8. Proteins 77 (Suppl 9) 114-122. (doi:10.1002/prot.22570)

Li Y, Willer CJ, Ding J, Scheet P \& Abecasis GR $2010 \mathrm{MaCH}$ : using sequence and genotype data to estimate haplotypes and unobserved genotypes. Genetic Epidemiology 34 816-834. (doi:10.1002/gepi.20533)

Margraf RL, Crockett DK, Krautscheid PM, Seamons R, Calderon FR, Wittwer CT \& Mao R 2009 Multiple endocrine neoplasia type 2 RET protooncogene database: repository of MEN2-associated RET sequence variation and reference for genotype/phenotype correlations. Human Mutation 30 548-556. (doi:10.1002/humu.20928)

Pęczkowska M, Kowalska A, Sygut J, Waligórski D, Malinoc A, JanaszekSitkowska H, Prejbisz A, Januszewicz A \& Neumann HP 2013 Testing new susceptibility genes in the cohort of apparently sporadic phaeochromocytoma/paraganglioma patients with clinical 
characteristics of hereditary syndromes. Clinical Endocrinology $\mathbf{7 9}$ 817-823. (doi:10.1111/cen.12218)

Plaza-Menacho I, van der Sluis T, Hollema H, Gimm O, Buys CH, Magee AI, Isacke CM, Hofstra RM \& Eggen BJ 2007 Ras/ERK1/2-mediated STAT3 $\mathrm{Ser}^{727}$ phosphorylation by familial medullary thyroid carcinomaassociated RET mutants induces full activation of STAT3 and is required for $c$-fos promoter activation, cell mitogenicity, and transformation. Journal of Biological Chemistry 282 6415-6424. (doi:10.1074/jbc.M608952200)

Plaza-Menacho I, Barnouin K, Goodman K, Martínez-Torres RJ, Borg A, Murray-Rust J, Mouilleron S, Knowles P \& McDonald NQ 2014 Oncogenic RET kinase domain mutations perturb the autophosphorylation trajectory by enhancing substrate presentation in trans. Molecular Cell 53 738-751. (doi:10.1016/j.molcel.2014.01.015)

Ren J, Wen L, Gao X, Jin C, Xue Y \& Yao X 2009 DOG 1.0: illustrator of protein domain structures. Cell Research 19 271-273. (doi:10.1038/cr.2009.6)

Rich TA, Feng L, Busaidy N, Cote GJ, Gagel RF, Hu M, Jimenez C, Lee JE, Perrier N, Sherman SI et al. 2014 Prevalence by age and predictors of medullary thyroid cancer in patients with lower risk germline RET proto-oncogene mutations. Thyroid 24 1096-1106. (doi:10.1089/thy.2013.0620)

Rückert F, Görgens H, Richter I, Krex D, Schackert G, Kuhlisch E, Fitze G, Saeger HD, Pilarsky C, Grützmann R et al. 2011 RET-protooncogene variants in patients with sporadic neoplasms of the digestive tract and the central nervous system. International Journal of Colorectal Disease 26 835-840. (doi:10.1007/s00384-011-1150-7)

Ruiz-Ferrer M, Fernández RM, Antiñolo G, López-Alonso M, Eng C \& Borrego S 2006 A complex additive model of inheritance for Hirschsprung disease is supported by both RET mutations and predisposing RET haplotypes. Genetics in Medicine 8 704-710. (doi:10.1097/01.gim.0000245632.06064.f1)

Seri M, Yin L, Barone V, Bolino A, Celli I, Bocciardi R, Pasini B, Ceccherini I, Lerone M, Kristoffersson U et al. 1997 Frequency of RET mutations in long- and short-segment Hirschsprung disease. Human Mutation 9 243-249. (doi:10.1002/(SICI) 1098-1004(1997)9:3 < 243::AIDHUMU5 > 3.0.CO;2-8)

Tamanaha R, Camacho CP, Ikejiri ES, Maciel RM \& Cerutti JM 2007 Y791F RET mutation and early onset of medullary thyroid carcinoma in a Brazilian kindred: evaluation of phenotype-modifying effect of germline variants. Clinical Endocrinology 67 806-808. (doi:10.1111/ j.1365-2265.2007.02964.x)
Toledo SP, dos Santos MA, Toledo RA \& Lourenço DM Jr 2006 Impact of RET proto-oncogene analysis on the clinical management of multiple endocrine neoplasia type 2. Clinics (Sao Paulo) 61 59-70. (doi:10.1590/ S1807-59322006000100011)

Toledo RA, Wagner SM, Coutinho FL, Lourenço DM Jr, Azevedo JA, Longuini VC, Reis MT, Siqueira SA, Lucon AM, Tavares MR et al. 2010 High penetrance of pheochromocytoma associated with the novel C634Y/Y791F double germline mutation in the RET protooncogene. Journal of Clinical Endocrinology and Metabolism 95 1318-1327. (doi:10.1210/jc.2009-1355)

Valente FO, Dias da Silva MR, Camacho CP, Kunii IS, Bastos AU, da Fonseca CC, Simião HP, Tamanaha R, Maciel RM \& Cerutti JM 2013 Comprehensive analysis of RET gene should be performed in patients with multiple endocrine neoplasia type 2 (MEN 2) syndrome and no apparent genotype-phenotype correlation: an appraisal of p.Y791F and p.C634Y RET mutations in five unrelated Brazilian families. Journal of Endocrinological Investigation 36 975-981. (doi:10.3275/8997)

Vestergaard P, Vestergaard EM, Brockstedt H \& Christiansen P 2007 Codon Y791F mutations in a large kindred: is prophylactic thyroidectomy always indicated? World Journal of Surgery 31 996-1001. (doi:10.1007/ s00268-006-0446-1)

Vierhapper H, Rondot S, Schulze E, Wagner L, Hanslik S, Niederle B, Bieglmayer C, Kaserer K \& Baumgartner-Parzer S 2005 Primary hyperparathyroidism as the leading symptom in a patient with a Y791F RET mutation. Thyroid 15 1303-1308. (doi:10.1089/thy. 2005.15.1303)

Virtanen VB, Pukkala E, Kivisaari R, Salo PP, Koivusalo A, Arola J, Miettinen PJ, Rintala RJ, Perola M \& Pakarinen MP 2013 Thyroid cancer and co-occurring RET mutations in Hirschsprung disease. Endocrine-Related Cancer 20 595-602. (doi:10.1530/ERC-13-0082)

Weber F \& Eng C 2005 Editorial: Germline variants within RET: clinical utility or scientific playtoy? Journal of Clinical Endocrinology and Metabolism 90 6334-6336. (doi:10.1210/jc.2005-2030)

Wells SA Jr, Gosnell JE, Gagel RF, Moley J, Pfister D, Sosa JA, Skinner M, Krebs A, Vasselli J \& Schlumberger M 2010 Vandetanib for the treatment of patients with locally advanced or metastatic hereditary medullary thyroid cancer. Journal of Clinical Oncology 28 767-772. (doi:10.1200/JCO.2009.23.6604)

Received in final form 24 November 2014

Accepted 25 November 2014

Made available online as an Accepted Preprint

25 November 2014 http://erc.endocrinology-journals.org

DOI: 10.1530/ERC-14-0491 (c) 2015 The authors Printed in Great Britain
Published by Bioscientifica Ltd. 